\title{
AIP
}

\section{First principles results on TbAl3(BO3)4: A promising magneto-optical material}

Y. Saeed, N. Singh, and U. Schwingenschlögl

Citation: Journal of Applied Physics 110, 103512 (2011); doi: 10.1063/1.3662176

View online: http://dx.doi.org/10.1063/1.3662176

View Table of Contents: http://scitation.aip.org/content/aip/journal/jap/110/10?ver=pdfcov

Published by the AIP Publishing

\section{Articles you may be interested in}

$(\mathrm{Sr}, \mathrm{Ba})(\mathrm{Si}, \mathrm{Ge}) 2$ for thin-film solar-cell applications: First-principles study

J. Appl. Phys. 115, 203718 (2014); 10.1063/1.4880662

Electronic structure and optical properties of $\mathrm{ZnSiO} 3$ and $\mathrm{Zn} 2 \mathrm{SiO} 4$

J. Appl. Phys. 106, 123701 (2009); 10.1063/1.3268445

First-principles study of the electronic and optical properties in rhombohedral LaAIO 3

J. Appl. Phys. 104, 053503 (2008); 10.1063/1.2973671

Optical properties of rocksalt and zinc blende AIN phases: First-principles calculations

J. Appl. Phys. 103, 073707 (2008); 10.1063/1.2903138

First-principles study of the structure, electronic, and optical properties of orthorhombic $\mathrm{Bi}$ In $\mathrm{O} 3$

Appl. Phys. Lett. 91, 071902 (2007); 10.1063/1.2770761

MIT LINCOLN

LABORATORY CAREERS

Discover the satisfaction of innovation and service

to the nation
- Space Control

- Air \& Missile Defense

- Communications Systems \& Cyber Security

- Intelligence, Surveillance and

Reconnaissance Systems

- Advanced
Electronics
- Tactical Systems
- Homeland
Protection
- Air Traffic Control

LINCOLN LABORATORY

MassachusetTs Institute of TeChNOLOgY

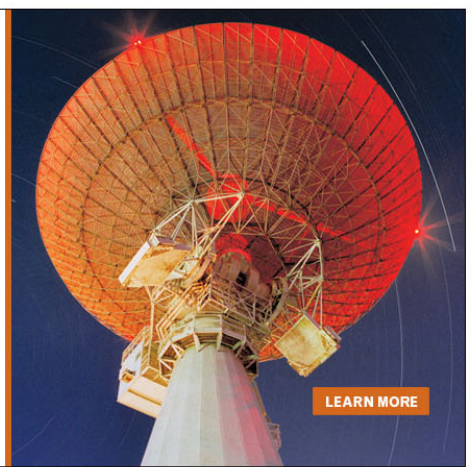




\title{
First principles results on $\mathrm{TbAl}_{3}\left(\mathrm{BO}_{3}\right)_{4}$ : A promising magneto-optical material
}

\author{
Y. Saeed, N. Singh, and U. Schwingenschlögl $\left.\right|^{\text {a) }}$ \\ KAUST, Physical Science and Engineering Division, Thuwal 23955-6900, Kingdom of Saudi Arabia
}

(Received 13 August 2011; accepted 12 October 2011; published online 21 November 2011)

\begin{abstract}
The electronic and optical properties of $\mathrm{TbAl}_{3}\left(\mathrm{BO}_{3}\right)_{4}$ are determined using density functional theory. The calculated total magnetic moment of $5.96 \mu_{B}$ is close to the expected moment of $\mathrm{Tb}^{3+}$. The frequency dependent dielectric function, refractive index, extinction coefficient, absorption, optical reflectivity, and energy loss function are explained in terms of the transitions between the valence and conduction bands. We find very high Kerr angles for ultraviolet light and potential for extension, even into the visible range by band structure design. (C) 2011 American Institute of Physics. [doi:10.1063/1.3662176]
\end{abstract}

\section{INTRODUCTION}

Borates are used in a vast number of applications, ranging from glass to fibers and flame retardants. Interestingly, a non-centrosymmetric geometry appears to be typical for borate structures. ${ }^{1}$ The compounds consequently have attracted much attention by outstanding (non-)linear optical properties, which are useful in magnetic devices for optical communication and measurement technology. ${ }^{2-5}$ Comparison of experimental as well as theoretical results for this class of materials indicates that the planar $\left[\mathrm{BO}_{3}\right]^{3-}$ group gives larger non-linear optical contributions than the tetrahedral $\left[\mathrm{BO}_{4}\right]^{5-}$ group. ${ }^{6}$ The Kerr and Faraday rotations in borate glasses have been addressed in Ref. 7 .

Borates can be doped by rare earth ions for using them as magneto-optical materials. ${ }^{8}$ The iron-based rare earth borates $\mathrm{RFe}_{3}\left(\mathrm{BO}_{3}\right)_{4}(\mathrm{R}=\mathrm{Nd}, \mathrm{Ho}, \mathrm{Gd}, \mathrm{Tb}$, and $\mathrm{Y})$ are interesting because of a large magneto-electric effect and non-centrosymmetric geometry, which makes them candidates for application in (non-)linear optical and magnetooptical devices. $^{9-18}$ In rare earth iron borates, the exchange interaction between the $\mathrm{Fe} 3 d$ electrons gives rise to antiferromagnetic ordering. Coupling of the $\mathrm{Fe}$ moments to the rare earth moments with a strong single-ion anisotropy results in complex magnetic phases, spin reorientation phase transitions, and (non-)collinear magnetism, as discovered by neutron scattering. ${ }^{19-22}$ The complexity of the magnetic structures associated with the $d$ and $f$ electrons makes it difficult to understand the microscopic details of the magnetoelectric effect. Moreover, Fe does not play an important role for the large magneto-electric effect in rare earth iron borates. $^{23}$

The magnetic complexity can be reduced by substituting $\mathrm{Fe}$ by $\mathrm{Al}$, which results in a new class of isostructural borates without $d$ valence electrons, $\mathrm{RAl}_{3}\left(\mathrm{BO}_{3}\right)_{4}$. A lot of experimental studies on the spectroscopic and optical properties of these materials have been performed, while a theoretical treatment is lacking in the literature. ${ }^{23-35}$ Recently,

\footnotetext{
a) Author to whom correspondence should be addressed. Electronic mail: udo.schwingenschlogl@kaust.edu.sa.
}

Chaudhury et $a .^{23}$ have studied the magneto-electricity and magneto-striction in $\mathrm{TmAl}_{3}\left(\mathrm{BO}_{3}\right)_{4}$ and concluded that the magneto-electricity is related to the piezoelectric effect of the non-centrosymmetric structure. The giant magnetoelectric effect in $\mathrm{HoAl}_{3}\left(\mathrm{BO}_{3}\right)_{4}$ has been found to increase with decreasing magnetic anisotropy. ${ }^{24}$ Moreover, the optical absorption edges of undoped and $\mathrm{Sm}^{3+}$ doped $\mathrm{YAl}_{3}\left(\mathrm{BO}_{3}\right)_{4}$ have been studied in the vacuum ultraviolet spectral region. $^{25}$

Since $\mathrm{TbAl}_{3}\left(\mathrm{BO}_{3}\right)_{4}$ has potential in laser applications, ${ }^{26-30}$ its optical absorption and luminescence have been measured by Kellendonk et $a .^{31}$ and later by Couwenberg et al., ${ }^{32}$ who found that the crystal field parameters are comparable with those of $\mathrm{Eu}^{3+}$-doped $\mathrm{GdAl}_{3}\left(\mathrm{BO}_{3}\right)_{4} \cdot{ }^{33}$ The structural and optical properties of $\mathrm{TbAl}_{3}\left(\mathrm{BO}_{3}\right)_{4}$ single crystals have been studied in Ref. 34. The $4 f^{n} \rightarrow 4 f^{n-1} 5 d$ absorption plays a central role for the optical and magneto-optical properties. $^{35}$

\section{METHODOLOGY}

In this work, we investigate the electronic structure of $\mathrm{TbAl}_{3}\left(\mathrm{BO}_{3}\right)_{4}$ and interrelate it with the optical and magnetooptical properties of the compound, due to its potential in (non-)linear optical devices. We study the band structure (BS) and density of states (DOS) in order to explain the optical conductivity and complex Kerr spectra in terms of electronic transitions. Our calculations are based on density functional theory, using the full-potential linearized augmented plane wave (FP-LAPW) method as implemented in the WIEN2k code. ${ }^{36}$ We have performed spin-polarized calculations, including spin-orbit coupling (SOC), with the magnetization along the (001) direction. We use the local spin density approximation plus onsite Coulomb interaction $(\mathrm{LSDA}+U)$ method and apply the exchange-correlation potential parameterization of von Barth and Hedin. ${ }^{37}$ This approach describes the ground state of the present compound with high accuracy. ${ }^{38,39}$ On the other hand, calculation of optical spectra, in principle, involves excited states. Thus, additional approximations have to be introduced, which, however, do not compromise the following line of reasoning, 
as they are only gradual and not qualitative. ${ }^{40}$ The generalized gradient approximation (GGA) is used to compare the results to the isostructural compound $\mathrm{TbFe}_{3}\left(\mathrm{BO}_{3}\right)_{4} \cdot{ }^{41}$

The crystal structure of $\mathrm{TbAl}_{3}\left(\mathrm{BO}_{3}\right)_{4}$ has a trigonal symmetry with space group R32 (No. 155). The cell parameters are $a=b=9.2933 \AA$ and $c=7.2496 \AA^{34}$ The O-B-O bond angle is $120^{\circ}$ and the $\mathrm{B}-\mathrm{O}$ bond distance is $1.383 \AA$. In the FP-LAPW method, the unit cell is divided into two parts: non-overlapping atomic spheres centered at the atomic sites and the interstitial region. The convergence parameter $R_{\operatorname{mt}} K_{\max }$, where $K_{\max }$ is the plane-wave cut-off and $R_{m t}$ is the smallest of all atomic sphere radii, controls the size of the basis. It is set to $R_{\operatorname{mt}} K_{\max }=7$ with $G_{\max }=18$. Convergence tests have shown that $63 \mathrm{k}$-points in the irreducible wedge of the Brillouin zone are sufficient, as more k-points give no appreciable change in energy or properties. A dense mesh of 174 uniformly distributed k-points is required in the optical calculations. Self-consistency is assumed for a total energy convergence of less than $10^{-5} \mathrm{Ry}$.

The LSDA $+U$ approach allows us to overcome the shortcoming of the LSDA that the Tb $4 f$ states appear at the Fermi level and are partially occupied, contradicting an atomic-like nature. A finite $U$ results in a Hubbard splitting of the occupied and empty $\mathrm{Tb} 4 f$ states. We use $U_{\text {eff }}=5 \mathrm{eV}$, as suggested in Ref. 42. Our LSDA $+U$ calculations yield a total magnetic moment of $5.96 \mu_{B}$, in good agreement with the $6 \mu_{B}$ expected for $\mathrm{Tb}^{3+}$ ions. A small amount of the magnetic moment $\left(0.05 \mu_{B}\right)$ comes from the interstitial region, while negligible contributions arise from $\mathrm{B}, \mathrm{O}$, and $\mathrm{Al}$ $\left(<0.004 \mu_{B}\right)$.

\section{RESULTS AND DISCUSSION}

The calculated LSDA $+U$ BS and DOS are shown in Figs. 1 and 2, respectively. We find $\mathrm{TbAl}_{3}\left(\mathrm{BO}_{3}\right)_{4}$ to be a wide bandgap insulator with a bandgap of $3.3 \mathrm{eV}$. The flat bands around $4 \mathrm{eV}$ are due to empty $\mathrm{Tb} 4 f$ states. These states are shifted deep inside the valence band as compared to the LSDA results (not shown). The DOS plots reveal the majority spin $\mathrm{Tb} 4 f$ states mainly between -7 and $-2 \mathrm{eV}$, while the minority spin states are almost empty, except for a sharp peak close to the Fermi energy. Below the Fermi energy, the valence band maximum mainly originates from the $\mathrm{O} 2 p$ states with small contributions of $\mathrm{B} 2 p$ and $\mathrm{Al} 3 p$, which are similar to those observed in other borates. ${ }^{46}$ The empty $\mathrm{Tb} 4 f$ states give rise to strong peaks for the minority spin, while the majority spin DOS is very small in this energy range (see the inset in Fig. 2). Above $5 \mathrm{eV}$, the unoccupied states are due to the Tb $5 d$ bands with small B $2 p$ and $\mathrm{Al} 3 p$ contributions. No appreciable changes appear in the electronic structure when $\mathrm{Fe}$ is replaced by $\mathrm{Al}$, except for the fact that the $\mathrm{Fe}$ $3 d$ states lie near the Fermi energy in the valence band of $\mathrm{TbFe}_{3}\left(\mathrm{BO}_{3}\right)_{4} \cdot{ }^{41}$ The energy level positions of the $\mathrm{Tb} 4 f$ states remain unchanged for GGA and GGA + SOC calculations. In the LSDA $+U$ approach, these states shift off the Fermi energy, as expected.

Comparison of our GGA results with calculations for $\mathrm{TbFe}_{3}\left(\mathrm{BO}_{3}\right)_{4}$ (Ref. 41) indicate that the ferroelectric polarization of $\mathrm{TbFe}_{3}\left(\mathrm{BO}_{3}\right)_{4}$ is largely due to the absence of

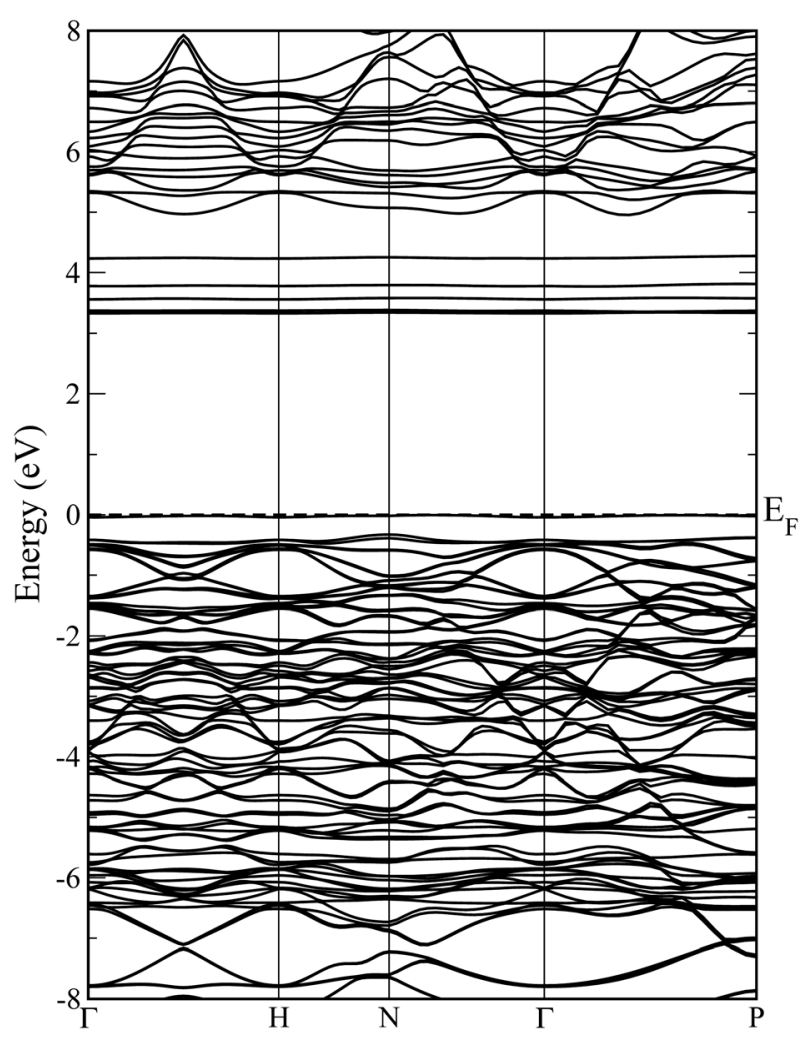

FIG. 1. BS of $\mathrm{TbAl}_{3}\left(\mathrm{BO}_{3}\right)_{4}$ as obtained from $\mathrm{LDA}+U$ calculations.

inversion symmetry in the crystal structure and is only weakly affected by the antiferromagnetic ordering. Therefore, Fe plays kind of no role for the optical and magnetooptical properties, but increases the magnetic complexity.

Figure 3 shows the calculated real, $\operatorname{Re}[\varepsilon]$, and imaginary, $\operatorname{Im}[\varepsilon]$, parts of the frequency-dependent dielectric function of $\mathrm{TbAl}_{3}\left(\mathrm{BO}_{3}\right)_{4}$. $\operatorname{Im}[\varepsilon]$ reveals a threshold energy of $3.3 \mathrm{eV}$ followed by two peaks at 8.5 and $15 \mathrm{eV}$, which are due to transitions from occupied $\mathrm{O} 2 p$ and $\mathrm{Tb} 4 f$ states to unoccupied $\mathrm{Tb} 5 d$ states. Furthermore, there are oscillating structures in the energy range from 10 to $15 \mathrm{eV}$, followed by a drop toward higher energy. $\operatorname{Re}[\varepsilon]$ shows a pronounced peak at $6.5 \mathrm{eV}$ and a shallow minimum around $17 \mathrm{eV}$ with a negative value. The structures around $18 \mathrm{eV}$ trace back to transitions between occupied $\mathrm{Tb} 4 f$ and unoccupied $\mathrm{Tb} 5 d$ states. They are not sensitive to $U$, since the onsite interaction influences only the Tb $4 f$ states. The static dielectric constant equals $\operatorname{Re}[\varepsilon(0)]=3.15$.

Figure 4 addresses the calculated frequency-dependent refractive index $n(\omega)$, extinction coefficient $k(\omega)$, absorption $\alpha(\omega)$, energy loss function $L(\omega)$, as well as reflectivity $R(\omega)$ of $\mathrm{TbAl}_{3}\left(\mathrm{BO}_{3}\right)_{4}$. We obtain $n(0)=1.78$, which is very close to the value (1.75) reported for $\mathrm{GdAl}_{3}\left(\mathrm{BO}_{3}\right)_{4}$ (Ref. 33) and, as expected, clearly less than found for $\mathrm{YAl}_{3}\left(\mathrm{BO}_{3}\right)_{4} \cdot{ }^{43,44} \mathrm{For}$ $n(\omega)$ and $k(\omega)$, we have maxima at 6 and $15.5 \mathrm{eV}$, respectively. The maximal reflectivity (minimal absorption) is found near $22 \mathrm{eV}$. The maximal absorption, on the other hand, occurs in the low energy range, due to the large number of bands just below the Fermi energy. A similar behavior has been reported for the absorption spectrum of $(\mathrm{Li} / \mathrm{Cs}) \mathrm{B}_{3} \mathrm{O}_{5}$ (see Ref. 45 and the references given therein). 

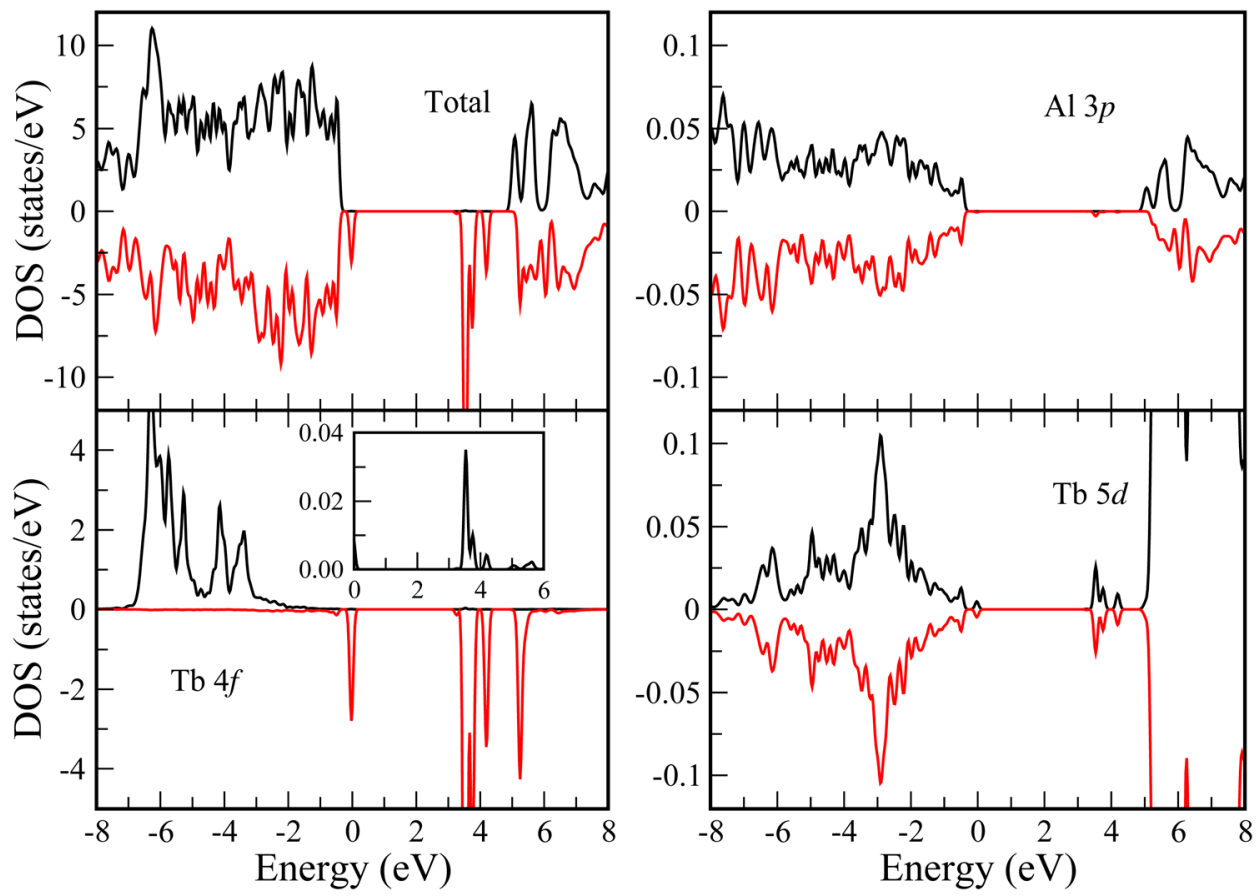

FIG. 2. (Color online) Total DOS and projected DOS of the $\mathrm{Al} 3 p, \mathrm{~Tb} 4 f$, and Tb $5 d$ orbitals separated in majority and minority spin contributions. The inset magnifies the majority spin $\mathrm{Tb} 4 f$ DOS from 0 to $6 \mathrm{eV}$.
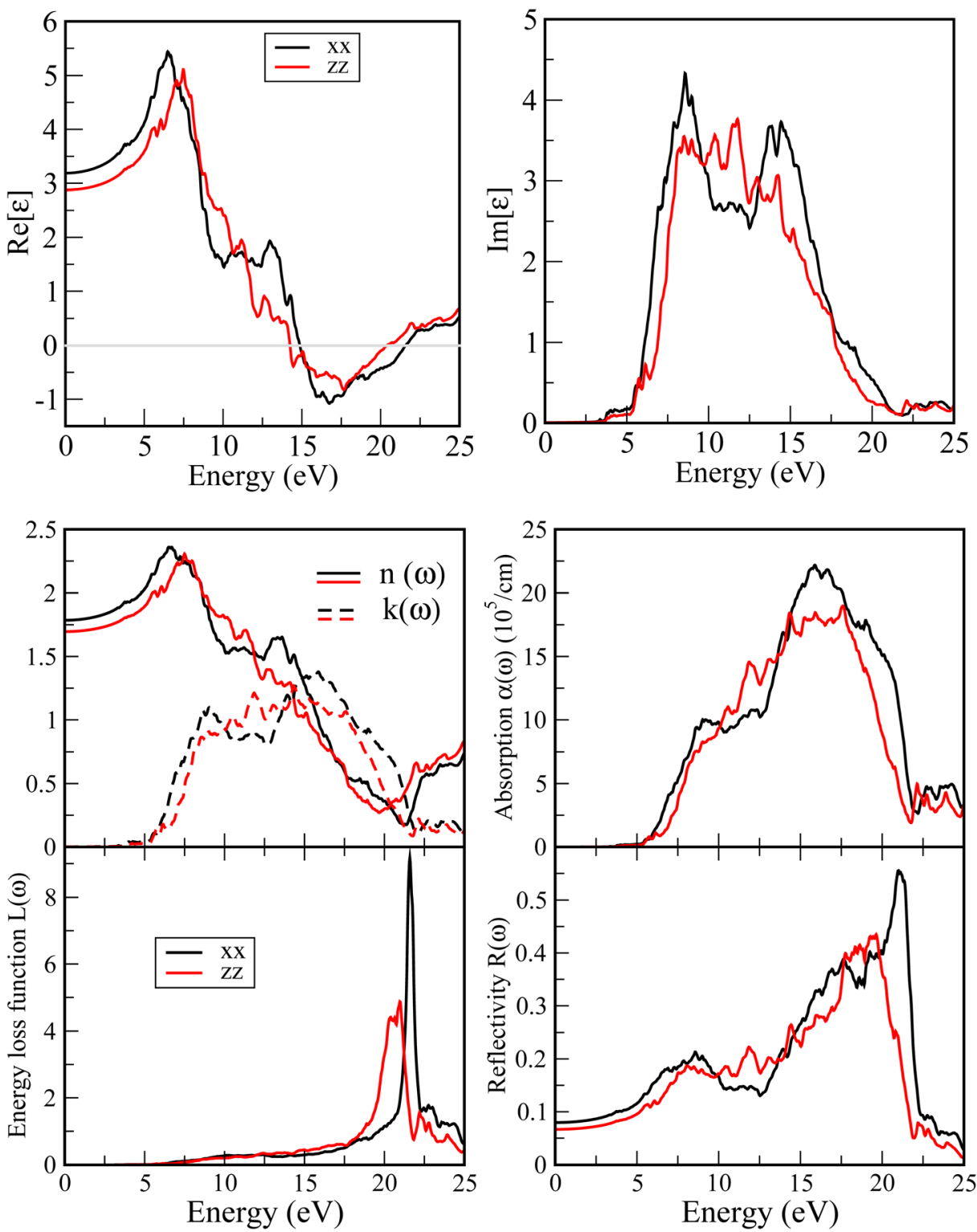

FIG. 3. (Color online) Real and imaginary components of the dielectric tensor of $\mathrm{TbAl}_{3}\left(\mathrm{BO}_{3}\right)_{4}$ along the perpendicular (xx) and parallel (zz) directions.

FIG. 4. (Color online) Refractive index $n(\omega)$, extinction coefficient $k(\omega)$, absorption $\alpha(\omega)$, energy loss function $L(\omega)$, and reflectivity $R(\omega)$. 

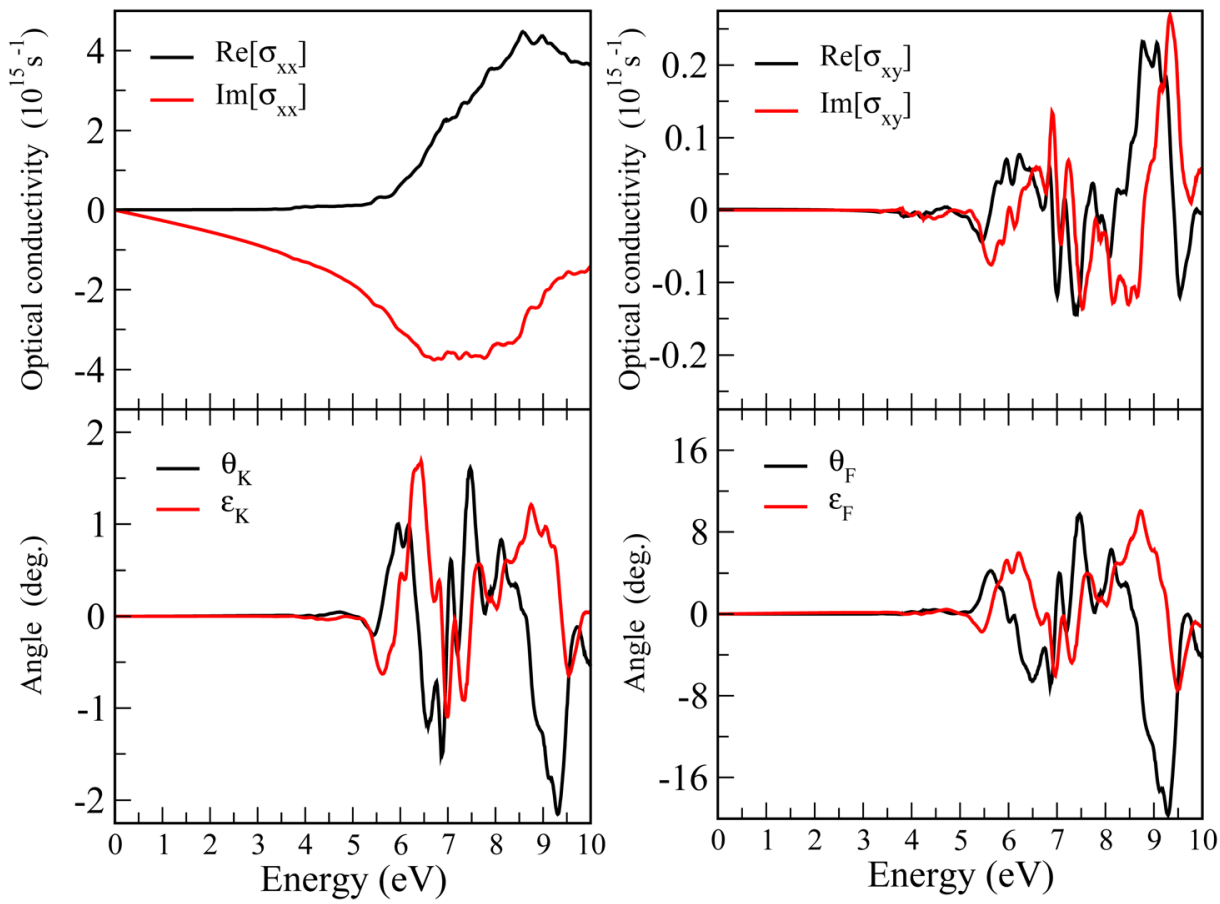

FIG. 5. (Color online) Diagonal $\left(\sigma_{x x}\right)$ and off-diagonal $\left(\sigma_{x y}\right)$ optical conductivities, Kerr rotation $\left(\theta_{K}\right)$ and ellipticity $\left(\varepsilon_{K}\right)$, as well as Faraday rotation $\left(\theta_{F}\right)$ and ellipticity $\left(\varepsilon_{F}\right)$
The energy loss of fast electrons traversing the material, $L(\omega)$, shows a distinct maximum near $22 \mathrm{eV}$, associated with plasma oscillations. At this energy, $\operatorname{Im}[\varepsilon]$ is small (see Fig. 3). The structures present in the optical spectra between 6 and $15.5 \mathrm{eV}$ originate mainly from transitions from the top of the valence band to unoccupied $\mathrm{Tb} 5 d$ states.

To understand the relationship between the optical conductivity and the Kerr spectra, we have calculated the magneto-optical properties, which have been measured by Sawannobori. ${ }^{47}$ The frequency-dependent diagonal and offdiagonal components of the optical conductivity tensor are shown in Fig. 5. A broadening of $0.1 \mathrm{eV}$ is employed to simulate the experimental finite lifetime. $\operatorname{Re}\left[\sigma_{x x}\right]$ and $\sigma_{x y}$ almost vanish up to $3.3 \mathrm{eV}$, which equals the energy gap, while $\operatorname{Im}\left[\sigma_{x x}\right]$ shows structures in the whole energy range. $\operatorname{Re}\left[\sigma_{x x}\right]$ exhibits a peak conductivity of $4.5 \times 10^{15} \mathrm{~s}^{-1}$ at $8.5 \mathrm{eV}$, while $\operatorname{Im}\left[\sigma_{x x}\right]$ shows a deep minimum around $7 \mathrm{eV} . \operatorname{Im}\left[\sigma_{x y}\right]$ has a maximum at $9.5 \mathrm{eV}$ and a minimum at $7.5 \mathrm{eV}$. $\operatorname{Re}\left[\sigma_{x y}\right]$, on the other hand, has a maximum at $9 \mathrm{eV}$ and a minimum at $7.5 \mathrm{eV}$. The structure present in the low energy range (around $4 \mathrm{eV}$ ) can be explained by transitions from $\mathrm{O} 2 p$ and $\mathrm{Al} 2 p$ to $\mathrm{Tb} 5 d$ states. The higher energy structures are due to transitions from $\mathrm{Tb} 4 f$ to $\mathrm{Tb} 5 d$ states.

The Kerr rotation $\theta_{K}$ and ellipticity $\varepsilon_{K}$ as well as the Faraday rotation $\theta_{F}$ and ellipticity $\varepsilon_{F}$ are displayed in Fig. 5. Both $\theta_{K}$ and $\varepsilon_{K}$ are characterized by various peaks above $5 \mathrm{eV}$. The maxima of $\theta_{K}\left(-2.1^{\circ}\right)$ and $\theta_{F}\left(-20^{\circ}\right)$ appear in the region where $\operatorname{Re}\left[\sigma_{x x}\right]$ and $\operatorname{Re}\left[\sigma_{x y}\right]$ have the largest magnitude. In contrast, $\varepsilon_{K}\left(1.6^{\circ}\right)$ and $\varepsilon_{F}\left(9^{\circ}\right)$ have pronounced peaks where $\operatorname{Im}\left[\sigma_{x x}\right]$ and $\operatorname{Im}\left[\sigma_{x y}\right]$ are maximal, respectively. Our results show that the magneto-optical Kerr and Faraday effects strongly depend on the diagonal and off-diagonal components of the optical conductivity tensor. The fact that $\operatorname{Re}\left[\sigma_{x x}\right]$ is directly linked to the optical transitions, i.e., the electronic states, is fruitful for magneto-optical engineering. The electronic structure can be tuned in such a way that the maximum of $\operatorname{Re}\left[\sigma_{x x}\right]$ shifts toward the visible region. This is achieved by alloying to reduce the energy gap. The observed energy dependence of $\theta_{K}$ and $\varepsilon_{K}$ makes $\mathrm{TbAl}_{3}\left(\mathrm{BO}_{3}\right)_{4}$ directly suitable for application in ultraviolet magnetooptical laser devices.

\section{CONCLUSION}

In conclusion, we have discussed first principles findings on $\mathrm{TbAl}_{3}\left(\mathrm{BO}_{3}\right)_{4}$ obtained from density functional theory. The $\mathrm{Tb}$ total magnetic moment is found to be $5.94 \mu_{B}$, in agreement with previous experiments and calculations. We observe a wide direct bandgap of $3.3 \mathrm{eV}$. The DOS shows that the $\mathrm{Tb} 4 f$ states lie deep inside the valence band, similar to $\mathrm{TbFe}_{3}\left(\mathrm{BO}_{3}\right)_{4}$. We have analyzed the frequency-dependent dielectric function $\varepsilon(\omega)$, refractive index $n(\omega)$, extinction coefficient $k(\omega)$, absorption $\alpha(\omega)$, energy loss function $L(\omega)$, and reflectivity $R(\omega)$ along the $a$ and $c$-axes. The static refractive index $n(0)$ is close to that of $\mathrm{GdAl}_{3}\left(\mathrm{BO}_{3}\right)_{4}$. The Kerr and Faraday rotations are very large, amounting to $-2.1^{\circ}$ and $-20^{\circ}$ at $\sim 9 \mathrm{eV}$ (ultraviolet), respectively. In particular, $\mathrm{TbAl}_{3}\left(\mathrm{BO}_{3}\right)_{4}$ paves the way for a systematic tailoring of the magneto-optical properties according to technological needs by suitable dopants.

\section{ACKNOWLEDGMENTS}

We thank KAUST research computing for providing the computational resources for this study.

${ }^{1}$ G. Heller, "A survey of structural types of borates and polyborates," in Topics in Current Chemistry (Springer, Berlin, 1986).

${ }^{2}$ D. A. Keszler, Curr. Opin. Solid State Mater. Sci. 1, 204 (1996).

${ }^{3}$ P. Becker, Adv. Mater. 10, 979 (1998).

${ }^{4}$ M. Knitel, New Inorganic Scintillators and Storage Phosphors for Detection of Thermal Neutrons (Delft University Press, Delft, 1998).

${ }^{5}$ C. Chen, N. Ye, J. Lin, J. Jiang, W. Zeng, and B. Wu, Adv. Mater. 11, 1071 (1999). 
${ }^{6}$ D. Xue, K. Betzler, H. Hesse, D. Lammers, Solid State Commun. 114, 21 (2000).

${ }^{7}$ D. Adamenko, V. Adamiv, I. Klymiv, and R. Vlokh, Ukr. J. Phys. Opt. 12, 1 (2011).

${ }^{8}$ A. S. Aleksandrovsky, A. V. Malakhovskii, V. N. Zabluda, A. I. Zaitsev, and A. V. Zamkov, J. Phys. Chem. Solids 67, 1908 (2006).

${ }^{9}$ J.-C. Joubert, W. B. White, and R. Roy, J. Appl. Crystallogr. 1, 318 (1968).

${ }^{10}$ A. K. Zvezdin, S. S. Krotov, A. M. Kadomtseva, G. P. Vorobev, Yu. F. Popov, A. P. Pyatakov, L. N. Bezmaternykh, and E. A. Popova, JETP Lett. 81, 272 (2005).

${ }^{11}$ S. S. Krotov, A. M. Kadomtseva, Y. F. Popov, G. P. Vorob'ev, A. V. Kuvardin, K. I. Kamilov, L. N. Bezmaternykh, and E. A. Popova, J. Magn. Magn. Mater. 300, e426 (2006).

${ }^{12}$ A. K. Zvezdin, A. M. Kadomtseva, S. S. Krotov, A. P. Pyatakov, Y. F. Popov, and G. P. Vorob'ev, J. Magn. Magn. Mater. 300, 224 (2006).

${ }^{13}$ F. Yen, B. Lorenz, Y. Y. Sun, C. W. Chu, L. N. Bezmaternykh, and A. N. Vasiliev, Phys. Rev. B 73, 054435 (2006).

${ }^{14}$ A. K. Zvezdin, G. P. Vorob'ev, A. M. Kadomtseva, Y. F. Popov, A. P. Pyatakov, L. N. Bezmaternykh, A. V. Kuvardin, and E. A. Popova, JETP Lett. 83, 509 (2006).

${ }^{15}$ R. P. Chaudhury, F. Yen, B. Lorenz, Y. Y. Sun, L. N. Bezmaternykh, V. L. Temerov, and C. W. Chu, Phys. Rev. B 80, 104424 (2009).

${ }^{16}$ A. M. Kalashnikova, V. V. Pavlov, R. V. Pisarev, L. N. Bezmaternykh, M. Bayer, and T. Rasing, JETP Lett. 80, 293 (2004).

${ }^{17}$ A. G. Gavriliuk, S. A. Kharlamova, I. S. Lyubutin, I. A. Troyan, S. G. Ovchinnikov, A. M. Potseluiko, M. I. Eremets, and R. Boehler, JETP Lett. 80, 426 (2004).

${ }^{18}$ P. Goldner, O. Guillot-Noël, J. Petit, M. Popova, and L. Bezmaternykh, Phys. Rev. B 76, 165102 (2007).

${ }^{19}$ P. Fischer, V. Pomjakushin, D. Sheptyakov, L. Keller, M. Janoschek, B. Roessli, J. Schefer, G. Petrakovskii, L. Bezmaternykh, V. Temerov, and D. Velikanov, J. Phys.: Condens. Matter 18, 7975 (2006).

${ }^{20}$ C. Ritter, A. Balaev, A. Vorontynov, G. Petrakovskii, D. Velikanov, V. Temerov, and I. Gudim, J. Phys.: Condens. Matter 19, 196227 (2007).

${ }^{21}$ C. Ritter, A. Vorontynov, A. Pankrats, G. Petrakovskii, V. Temerov, I. Gudim, and R. Szymczak, J. Phys.: Condens. Matter 20, 365209 (2008).

${ }^{22}$ H. Mo, C. S. Nelson, L. N. Bezmaternykh, and V. T. Temerov, Phys. Rev. B 78, 214407 (2008).

${ }^{23}$ R. P. Chaudhury, B. Lorenz, Y. Y. Sun, L. N. Bezmaternykh, V. L. Temerov, and C. W. Chu, Phys. Rev. B 81, 220402 (2010).

${ }^{24}$ K.-C. Liang, R. P. Chaudhury, B. Lorenz, Y. Y. Sun, L. N. Bezmaternykh, V. L. Temerov, and C. W. Chu, Phys. Rev. B 83, 180417 (2011).
${ }^{25}$ V. P. Dotsenko, I. V. Berezovskaya, N. P. Efryushina, A. S. Voloshinovskii, and G. B. Stryganyuk, J. Mater. Sci. 45, 1469 (2010).

${ }^{26}$ J. Yasi, M. J. Myers, and D. Rhonehouse, Chin. J. Lasers (EE) 1(6), 513 (1992).

${ }^{27}$ T. Katsuhisa, H. Kazuyuk, and S. Naohiro, Jpn. J. Appl. Phys. 34, 4825 (1995).

${ }^{28}$ V. Jubera, J. P. Chaminade, A. Garcia, F. Guillen, and C. Fouassier, J. Lumin. 101, 1 (2003).

${ }^{29}$ K. Binnemans, R. Van Deun, C. Görller-Walrand, and J. L. Adam, J. NonCryst. Solids 238, 11 (1998).

${ }^{30}$ M. Nogami, T. Yamazaki, and Y. Abe, J. Lumin. 78, 63 (1998).

${ }^{31}$ F. Kellendonk and G. Blasse, Phys. Chem. Solids 43, 481 (1982).

${ }^{32}$ I. Couwenberg, K. Binnemans, H. De Leebeeck, and C. Görller-Walrand, J. Alloys Compd. 274, 157 (1998).

${ }^{33}$ C. Görller-Walrand, E. Huygen, K. Binnemans, and L. Fluyt, J. Phys. Condens. Matter 6, 7797 (1994).

${ }^{34}$ J. Lu, C. Fu, and J. Chen, Appl. Opt. 50, 1 (2011).

${ }^{35}$ C. B. Rubinstein, S. B. Berger, L. G. Van Uitert, and W. A. Bonner, J. Appl. Phys. 35, 2338 (1964).

${ }^{36}$ P. Blaha, K. Schwarz, G. Madsen, D. Kvasicka, and J. Luitz, WIEN2k: An Augmented Plane Wave Plus Local Orbitals Program for Calculating Crystal Properties (Vienna University of Technology, Vienna, 2001).

${ }^{37}$ U. von Barth and L. Hedin, J. Phys. C 5, 1629 (1972).

${ }^{38}$ U. Schwingenschlögl and C. Schuster, Phys. Rev. Lett. 99, 237206 (2007); EPL 79, 27003 (2007)

${ }^{39}$ U. Schwingenschlögl, C. Schuster, and R. Frésard, EPL 88, 67008 (2009); EPL 81, 27002 (2008)

${ }^{40}$ N. Singh and U. Schwingenschlögl, Chem. Phys. Lett. 508, 29 (2011); S. Nazir, N. Singh, and U. Schwingenschlýgl, Phys. Rev. B 83, 113107 (2011).

${ }^{41}$ C. Lee, J. Kang, K. H. Lee, and M. H. Whangbo, Chem. Mater. 21, 2534 (2009).

${ }^{42}$ J. K. Lang, Y. Baer, and P. A. Cox, J. Phys. F: Met. Phys. 11, 121 (1981).

${ }^{43}$ R. He, Z. S. Lin, M.-H. Lee, and C. T. Chen, J. Appl. Phys. 109, 103510 (2011).

${ }^{44}$ V. V. Filippov, I. T. Bondar, N. V. Kuleshov, N. I. Leonyuk, V. V. Maltsev, and O. V. Pilipenko, J. Opt. Technol. 74, 717 (2007).

${ }^{45}$ J. Li, C.-G. Duan, Z.-Q. Gu, and D.-S. Wang, Phys. Rev. B 57, 6925 (1998).

${ }^{46}$ Y. Wang, L. Wang, and H. Li, J. Appl. Phys. 102, 013711 (2007).

${ }^{47}$ N. Sawanobori, New Glass 18, 5 (2003). 